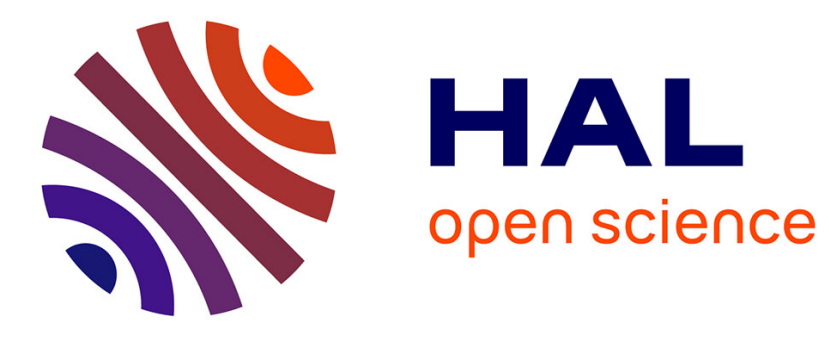

\title{
Impact of Machine's Robotisation on the Activity of an Operator in Picking Tasks
}

\author{
Adrian Couvent, Matthieu Dridi, Nicolas Tricot, Christophe Debain, \\ Mahmoud Almasri, Gil de Sousa, Gérard Chalhoub, Marie Izaute, Fabien \\ Coutarel
}

\section{To cite this version:}

Adrian Couvent, Matthieu Dridi, Nicolas Tricot, Christophe Debain, Mahmoud Almasri, et al.. Impact of Machine's Robotisation on the Activity of an Operator in Picking Tasks. 1st International Conference on Human Systems Engineering and Design: Future Trends and Applications (IHSED2018), Oct 2018, Reims, France. pp.387-393. halshs-01906631

\section{HAL Id: halshs-01906631 \\ https://shs.hal.science/halshs-01906631}

Submitted on 16 May 2020

HAL is a multi-disciplinary open access archive for the deposit and dissemination of scientific research documents, whether they are published or not. The documents may come from teaching and research institutions in France or abroad, or from public or private research centers.
L'archive ouverte pluridisciplinaire HAL, est destinée au dépôt et à la diffusion de documents scientifiques de niveau recherche, publiés ou non, émanant des établissements d'enseignement et de recherche français ou étrangers, des laboratoires publics ou privés. 
Author-produced version of the article published in Human Systems Engineering and Design. IHSED 2018. Advances in Intelligent Systems and Computing, vol. 876, p. 387-393

The original publication is available at

DOI: 10.1007/978-3-030-02053-8_59

\title{
Impact of machine's robotisation on the activity of an operator in picking tasks
}

\author{
Adrian Couvent ${ }^{1}$, Mathieu Dridi ${ }^{2}$, Nicolas Tricot $^{1}$, Christophe Debain $^{1}$, \\ Mahmoud Almasri ${ }^{1}$, Gil De Sousa ${ }^{1}$, Gerald Chaloub ${ }^{2}$, Marie Izaute ${ }^{3}$, Fabien Coutarel ${ }^{2}$ \\ ${ }^{1}$ IRSTEA, TSCF, Avenue Blaise Pascal. 9, \\ 63170 Aubière, France \\ \{adrian.couvent, nicolas.tricot, christophe.debain, mahmoud.almasri, \\ gil.de-sousa\}@irstea.fr \\ ${ }^{2}$ University Clermont Auvergne, \\ \{mathieu.dridi, gerald.chaloub, fabien.coutarel\}@uca.fr \\ ${ }^{3}$ LAPSCO, \\ \{marie.izaute\}@uca.fr
}

\begin{abstract}
This paper presents the results of an analysis of the activities during manual and robotised piece picking tasks to know and understand the impact of the robotisation. Here, this task is manually realized first and then partially automated with a robot. The activity is described with three indicators. These indicators are computed with image processing and a subdivision of the picking area. The robot has an impact on the activity because its introduction induces a convergence of the subjects to the same activity regardless without of their uses of technologies considering the interaction in this case.
\end{abstract}

Keywords: User-centred Systems · Human-Machine Systems · Usability and User Experience

\section{Introduction}

The human-machine interaction (HMI) is a key point for a suitable integration. Currently more and more machines need autonomy to work in team with human operators and other robots. Robots can also assist or work with other robots and humans. Therefore, Human-Robot Interaction (HRI) receives considerable attention because humans and robots are increasingly cooperating in many fields (transport, agriculture, industrial field...). Human-computer interaction (HCI) and HRI differ in several dimensions. HRI differs from HCI and HMI because it concerns systems which have complex and dynamic, control systems, show autonomy and cognition, and which operate in dynamic world as in [1] and [2]. 


\section{Background}

Regarding the teamwork between robots and humans (seen as agents), it seems important to have an efficient task repartition. It is noted in [3] that human skills are required in robotic systems. The performance approach to evaluate skills level, is used for robot assisted surgery [4] where the learning and the progress of skills are estimated with the performance of the surgeon. In [5], the comparison between manual and assisted work permits to estimate the progress of skills. Another approach is the use of mental models approach like in [6]. Skills estimation is a key to obtain an efficient task repartition.

The convergence of automated fields together with human sciences and the usercentred design described in [7] allows a machine adaptation to the human, the leverage of human skills and capacities, and to answer to the human failure. Many tasks are feasible with human-robot teams. In this situation the robot must understand the operator, as in [8], and adapts its activity. Many interesting surveys have studied the efficiency of human-robot teams as in [9]. But in the HRI, the efficiency is linked to the potential activities of each agents like described in [3]. Regarding this approach, it is interesting to understand the operator activity evolution without considering the performance. It is important to understand if the robot has an impact on the activity of the operator. To have an efficient HRI and a good acceptability, the robot must not impact the activity but must complete it. The workflow study is important to understand the force and the problem of the HRI as in [10].

It is proved in game theory that the behaviour of the user is influenced by his belief learning [11]. Regarding this, it is interesting to compare if the operator knowledge and technology uses have an influence in the HRI.

Considering the previous element with user-centred design in robotic, we hope that it is conceivable to have HRI which do not affect the activity. Regarding this, it is interesting to evaluate if, how and why the robotisation of machines affects the activity of operator.

\section{$3 \quad$ Field Test}

In order to evaluate if, how and why the robotisation of machines affects the activity of operator, we propose here to compare activity with and without robot thanks to a brief and typical example of picking task.

This comparison is achieved after a familiarization phase to get a repeatable and repeated activity. We suppose that this phase is completed when the operators have the same activity to do the same task twice. In this task of picking, the executed strategy is represented by the activity. In addition, another assumption is that the interaction level with the robot is linked with the use of technologies.

Considering these elements, an experiment has been developed to evaluate our hypotheses in the case of piece picking. For this purpose, an experimental platform has been designed in laboratory to evaluate the impact of a mobile robot to carry packages. 


\subsection{Experimental platform and robot}

The experimental platform is composed of four shelves, identified with letters. On each shelf, there are six packages, identified with the letter of the shelf and a number according to their position on the shelf. The lateral distance between each shelf is one meter and the shelves measure five-meters long.

To evaluate the impact of the robotisation, a cart is used first to establish and end the familiarization phase. The cart and the robot have the same sizes. The robot is the Sherpa by Norcan (depicted in Fig. 1). The robot is able to follow or precede someone thanks to a rangefinder and four other lidars to secure its displacement. The robot interface is composed of two set of four buttons, located at the front and at the rear. Each set is composed of two buttons to choose the mode: follow or precede mode. There is also one button to activate the automated return to the end point, and a red emergency button.

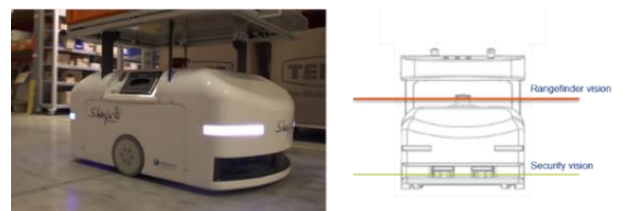

Fig. 1. Sherpa robot by Norcan with its description of element of vision.

\subsection{Experiment}

The experiment begins with a questionnaire about the use of technologies (smartphones, cars, smart homes and their connections). After an explanation of the task, the operator picks up ten orders of five packages randomly chosen with the cart. Between each order, the operator makes an audio recorded self-confrontation. After an introduction of the robot, the operator must pick up four packages and ten orders with the robot as in the previous step. During this experiment, all orders are recorded from a camera over the area. Audio recordings are realized for every self-confrontation. Recordings are also synchronized with data of the robot. The panel of subjects is composed of twenty-three voluntary people not familiar with picking tasks to ensure the observation of the phase of familiarization.

\section{Definition and analysis of activity}

To compare the activities of each subject, indicators have been designed. These indicators represent activities of subjects and are independent from the orders. They are used with a program and are based on the subdivision of the picking area depicted in Fig. 3.

The first indicator describes the independence between the subject and the cart. It means the time rate when the cart is in another area than the subject. In (1), $T_{\text {initial }}$ is the date at $\mathrm{t}=0 \mathrm{~s}$ and $T_{\text {final }}$ is the final date.

$$
I_{1}=\frac{\sum \text { Time }_{\text {subject and cart in different area }}}{T_{\text {final }}-T_{\text {initial }}} .
$$


The second indicator describes the method to pick up packages on shelves. This indicator highlights if the subject picks up packages on one shelf or two shelves at the same time.

$$
I_{2}=\frac{\text { Number of area where packages area picked up }}{\text { Number of shelves with packages which are collected }} .
$$

The third indicator describes if the subject pushes or pulls the cart/the robot.

$$
I_{3}=\frac{\sum_{i=0}^{\text {Nbrtransition }} \delta_{i}}{\text { Number of transition pass by the subject }} \text { with } \delta=\left\{\begin{array}{l}
1 \text { if the subject push } \\
0 \text { if the subject pull }
\end{array} .\right.
$$

A transition is the passage between two subdivisions. Regarding these three indicators it is possible to know the applied strategy. For example if $I_{1}=0, I_{2}=1$ and $I_{3}=1$, the subject pushes and stays with the cart and picks up packages shelf by shelf.

Thanks to these indicators, the end of the familiarization phase is recognized when they describe the same strategy twice or more. For example in Fig. 2, the familiarization phase is finished at the sixth order. After this order, the subject applies the same strategy for every order. The subject leaves his cart $\left(I_{1}>0.4\right)$, picks up package on two shelves in the same area $\left(I_{2}<1\right)$ and pushes the cart $\left(I_{3}=1\right)$.

Using this definition of the indicators, it is also possible to automate their computation. To do so, a program has been developed to generate automatically the subdivision of the picking area as shown in Fig. 3. This subdivision is based on an image processing of the video recorded during the experiment.

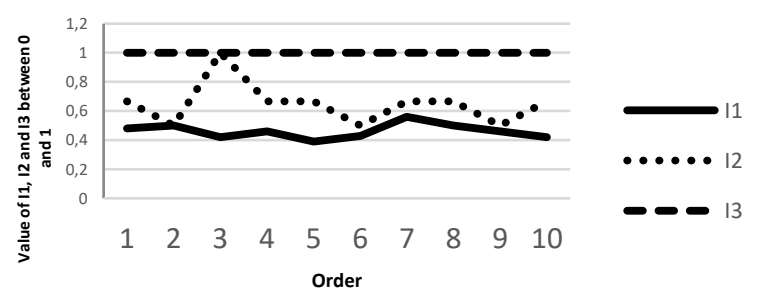

Fig. 2. Subject 15 indicators evolution during ten orders.

The program tracks the subject and detects the cart/robot thanks to theirs color and movement. Then, successive positions are recorded and the indicators are computed at the end of the video. The program gives two results, an image (Fig. 3) and csv files where indicators and positions are stored. The program computes the three indicators but currently the image processing need to be improved to avoid luminosity problems and false movements detection. To obtain a good description of the strategies chosen by the subjects, the proposed indicators are converted to binaries values. So for $I_{1}$, if the value is superior to 0.15 , the operator have the will to leave the cart. Therefore beyond this value, $I_{1}=1$ else $I_{1}=0$. In the case of $I_{2}$, if the value is equal to $1, I_{2}=1$ else $I_{2}=0$. For $I_{3}$, if the value is superior to 0.5 , the operator mostly pushes so $I_{3}=1$ else $I_{3}=0$. There are eight possible strategies. To quickly identify the strategy, $I_{1}$ is multiplied by $1, I_{2}$ by 10 and $I_{3}$ by 100 . The addition directly highlights the strategy applied 
by the subject. With this method, the hundred represent the value $I_{3}$, the tens the value of $I_{2}$ and the unities the value of $I_{1}$.

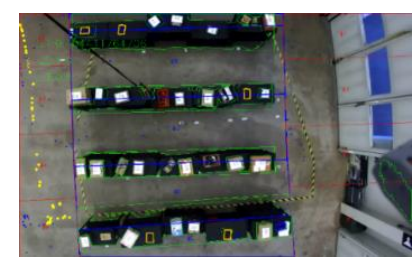

Fig. 3. Image result from the program with indicator. Subdivision of the area with blue centrals area and red borders area. Blue points are operator position and yellow cart position.

More than $90 \%$ of subjects applied the same strategy at the end of the experiment when using the robot. But it is interesting to compare the results of the questionnaire with the results of the experiment. In this purpose, an indicator, $\mathrm{NbComm}$, is created to estimate the number of collected orders before the end of the familiarization phase. Different variables regarding the use of technologies, smartphones $\mathrm{Sm}$, car Car and domotic Domo, are extracted from the questionnaire. The variable concerning the use of smartphones is composed of four modalities: no smartphones (Sm0), simple use $(\mathrm{SmP})$, classical use $(\mathrm{SmM})$ and intensive use $(\mathrm{SmF})$. The variables Car and Domo are composed of three modalities: no car or domotic (Car0 and Domo0), classical use (CarM and DomoM) and extensive use (CarF and DomoF). Regarding NbComm, this variable is composed of three modalities: need 1,2 or 3 orders to end the familiarization (NbCommP), need more than 3 and less than 6 orders to be familiarized (NbCommM) and need more than 6 orders (NbCommG). Based on these variables and their modalities, a Multiple Correspondence Analysis is achieved with result in Fig. 4.

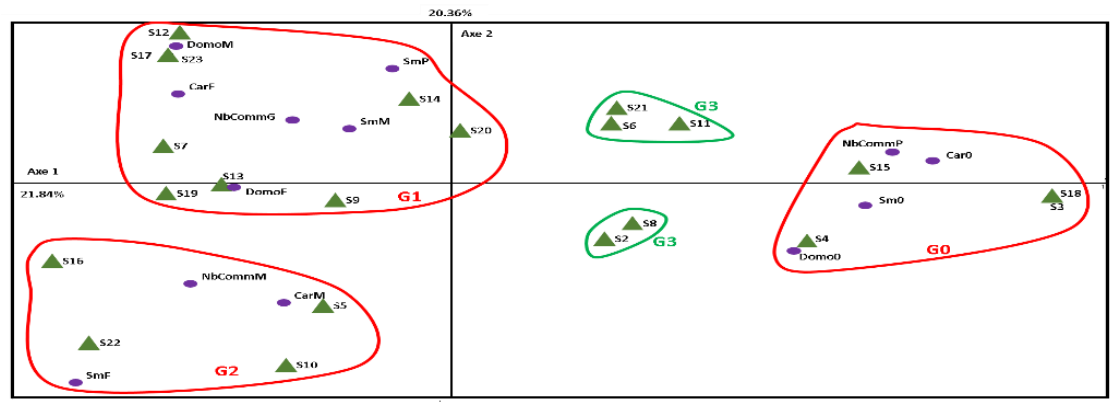

Fig. 4. Result of MCA projected on axes 1 (inertia of 21.84\%) and 2 (inertia of 20.36\%).

The MCA distinguishes three groups based on the use of the car, smartphones, domotic and the number of orders to be familiarized. Subjects without smartphones, car and domotic rapidly finish their familiarization (group G0 in red). Subjects who intensively use each technologic elements (G2), finish their familiarization between three and six orders. Subjects who classically use technologic elements (G1) finish their familiarization after six orders. The group (group in green) are subjects with an intensive use of technology who rapidly finish their familiarization. Three patterns of users can be observed here. 


\section{Conclusion}

In this paper, the impact of robotisation on the operator's activity is evaluated. It is clear that the proposed function of the robot (follow mode) influences the strategy of operators. To reduce this, a user-centered design of the function is essential. Another point discussed here is the linked between the use of technology and the interaction level. It is observed that subjects who intensively use technology need more orders to achieve their familiarization than subjects who have a classical use of technology.

The robotisation influences operators who choose the easiest way of use. Considering the link between the use of technology and the interaction level, more the subject is comfortable with the use of technology more he will interact with the robot.

\section{Acknowledgment}

This research was financed by the French government IDEX-ISITE initiative 16IDEX-0001 (CAP 20-25 with the support of the regional council Auvergne-RhôneAlpes and the support with the European Union via the program FEDER.

\section{$7 \quad$ References}

[1] T. Fong, C. Thorpe, et C. Baur, " Collaboration, Dialogue, Human-Robot Interaction », in Robotics Research, Springer, Berlin, Heidelberg, 2003, p. 255-266.

[2] M. A. Goodrich et A. C. Schultz, « Human-robot Interaction: A Survey », Found Trends Hum-Comput Interact, vol. 1, no 3, p. 203-275, janv. 2007.

[3] J. Scholtz, « Theory and Evaluation of Human Robot Interactions », janv. 2003.

[4] V. Chandra et al., "A comparison of laparoscopic and robotic assisted suturing performance by experts and novices », Surgery, vol. 147, n 6, p. 830-839, juin 2010.

[5] L. Chang, R. M. Satava, C. A. Pellegrini, et M. N. Sinanan, « Robotic surgery: identifying the learning curve through objective measurement of skill », Surg. Endosc. Interv. Tech., vol. 17, no 11, p. 1744-1748, nov. 2003.

[6] J. Graham, L. Zheng, et C. Gonzalez, « ACognitive Approach to Game Usability and Design: Mental Model Development in Novice Real-Time Strategy Gamers », Cyberpsychology Behav. Impact Internet Multimed. Virtual Real. Behav. Soc., vol. 9, p. 361-6, juill. 2006.

[7] C. Abras, D. Maloney-Krichmar, et J. Preece, « 1. Introduction and History », p. 14, 2004.

[8] S. Nikolaidis, D. Hsu, et S. Srinivasa, « Human-robot mutual adaptation in collaborative tasks: Models and experiments », Int. J. Robot. Res., vol. 36, nº 5-7, p. 618-634, juin 2017.

[9] M. C. Gombolay, R. A. Gutierrez, S. G. Clarke, G. F. Sturla, et J. A. Shah, « Decisionmaking authority, team efficiency and human worker satisfaction in mixed human-robot teams », Auton. Robots, vol. 39, n 3, p. 293-312, oct. 2015.

[10] J. L. Casper et R. R. Murphy, « Workflow study on human-robot interaction in USAR », in Proceedings 2002 IEEE International Conference on Robotics and Automation (Cat. No.02CH37292), 2002, vol. 2, p. 1997-2003 vol.2. 
[11] C. A. Ioannou et J. Romero, «A generalized approach to belief learning in repeated games », Games Econ. Behav., vol. 87, p. 178-203, sept. 2014. 